\title{
Performance Evaluation of a Stereoscopic Based 3D Surface Localiser for Image-Guided Neurosurgery
}

\author{
Perrine Paul, Oliver Fleig, Sabine Tranchant, and Pierre Jannin \\ Laboratoire IDM, Faculté de Médecine, Université de Rennes, 35043 Rennes, France \\ \{Perrine.Paul, Pierre. Jannin\}@univ-rennes1.fr
}

\begin{abstract}
This paper reports the performance evaluation of a method for visualisation and quantification of intraoperative cortical surface deformations. This method consists in the acquisition of $3 \mathrm{D}$ surface meshes of the operative field directly in the neuronavigator's coordinate system by means of stereoscopic reconstructions, using two cameras attached to the microscope oculars. The locations of about 300 surfaces are compared to the locations of two reference surfaces from a physical phantom: a segmented CT scan with image-to-physical fiducial-based registration, used to compute the overall system performance, and a cloud of points acquired with the neuronavigator's optical localiser, used to compute the intrinsic error of our method. The intrinsic accuracy of our method was shown to be within $1 \mathrm{~mm}$.
\end{abstract}

\section{Introduction}

Limitations of image-guided neurosurgery systems mainly concern (1) intraoperative visualisation of preoperative data and (2) intraoperative anatomical deformations. Concerning visualisation, most neuronavigation systems offer display of preoperative images in microscope oculars as 2D monochromatic contours whereas preoperative images are 3D and multimodal. Augmented reality with 3D graphics has been proposed by [1] but only for displaying the 3D surface of the lesion. Extending this approach to multimodal images raises the issue of how to display images without obstructing the normal vision of the field of view (FOV). Intraoperative deformations, due to both brain shift and resection, need to be detected in order to update preoperative images. The proposals found in the literature tend to favour the use of surface or volume intraoperative imagery. To account for these limitations, we previously proposed to map preoperative images to direct light images of the intraoperative FOV [2] in the virtual world.We called this approach augmented virtuality. We then applied stereoscopic reconstruction techniques to this approach, in order to acquire intraoperative 3D surface data sets 3]. We call this system a 3D surface localiser. Our approach was initially limited to visualisation features. Our medium term objective is to use this method to quantify the cortical surface shift. Similar proposals, using images acquired by cameras fixed to the surgical microscope, 
have been developed by 4] and [5]. In [6, a laser grid was projected onto the brain surface for surface deformation tracking purposes.

In this paper, we focus on evaluating the performance of our 3D surface localiser using a bone skull phantom. We first briefly summarise the principle of the 3D surface localiser. Two procedures of accuracy testing on a phantom are then described using a standardised framework [7]. The influence of certain input parameters is also evaluated. Lastly, the results obtained from 156 and 292 reconstructed surface meshes are provided and discussed.

\section{Material and Methods}

\subsection{D Surface Localiser}

The method used has already been described in [3]. Briefly, we used a standard neuronavigation system with a pair of stereo cameras attached to the surgical microscope. Colour images were taken alternately from the left and right oculars. A strong calibration process, which was initialised with the results of Tsai's camera calibration, allowed rectification of image pairs and the use of epipolar geometry. Point mapping based on zero-mean normalised sum of squared differences (ZNSSD) of luminance and epipolar geometry provided a disparity map which was used to reconstruct a surface mesh [8]. Owing to microscope position tracking by the neuronavigator, the reconstructed surface mesh was directly expressed into the neuronavigator's coordinate system.

\subsection{Performance Evaluation}

The performance evaluation procedures were based on a comparison with two different reference surfaces. The first (see Figure1), used by the FIDSCAN procedure, was the 3D surface segmented from a CT scan of a skull phantom registered to the neuronavigator's coordinate system by means of attached markers. The second reference surface (see Figure 1), used by the LOCCLOUD procedure, was a cloud of points acquired by the pointer of the optical localiser of the neuronavigation system, referred to as the pointer. Reconstructed surface meshes and reference surfaces are in the same coordinate system, that of the neuronavigator. The FIDSCAN procedure was used to study the overall performance of the $3 \mathrm{D}$ surface localiser including image-to-physical registration error. The LOCCLOUD procedure was used to measure the error due to the calibration and reconstruction method alone. A standardised framework, as proposed in [7], was applied to describe both procedures (see Table 1).

Validation data sets: For the two validation procedures, the same bone skull phantom (referred to as $D_{I}$ in Table 1) was used. 

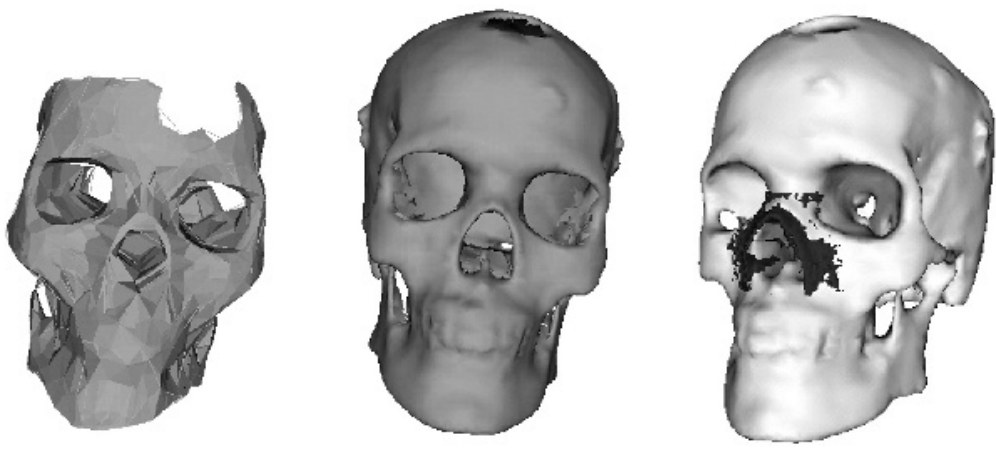

Fig. 1. Left, Reference 2, i.e., surface from the points cloud. Centre, Reference 1, i.e., surface of the CT scan. Right, a reconstructed surface mesh was superposed on Reference 1 (on the nose).

Table 1. Description of the two validation procedures

\begin{tabular}{|c|c|c|}
\hline & FIDSCAN & LOCCLOUD \\
\hline$\underline{D_{I}: \text { Validation data sets }}$ & Synthetic skull & Synthetic skull \\
\hline$P_{I}:$ Input parameters & FOV type, Light, Focus & FOV type, Light, Focus \\
\hline$F_{M}:$ Method to be evaluated & Surface localiser & Surface localiser \\
\hline$\hat{S}_{M}:$ Surfaces computed by $F_{M}$ & 292 meshes & 156 meshes \\
\hline $\begin{array}{l}F_{R}: \text { Function which computes } \\
\text { reference from } D_{I} \& P_{I}\end{array}$ & $\begin{array}{l}\mathrm{CT} \text { scan with fiducial } \\
\text { registration }\end{array}$ & $\begin{array}{l}\text { Points cloud acquired with } \\
\text { pointer }\end{array}$ \\
\hline $\begin{array}{l}\hat{S}_{R}: \text { Output data from } F_{R} \\
\text { (i.e., reference) }\end{array}$ & $\begin{array}{l}\text { CT scan volume in } \\
\text { neuronavigator's } \\
\text { coordinates system }\end{array}$ & $\begin{array}{l}2000 \text { points in } \\
\text { neuronavigator's } \\
\text { coordinates system }\end{array}$ \\
\hline $\begin{array}{l}\hat{E}_{R}: \text { Estimated error } \\
\text { relative to the computation } \\
\text { of } \hat{S}_{R} \text { by } F_{R}\end{array}$ & TRE & $\begin{array}{l}\text { Pointer } \\
\text { precision }\end{array}$ \\
\hline $\begin{array}{l}F_{N S R}: \text { Function which } \\
\text { transforms } \hat{S}_{R} \text { for comparison }\end{array}$ & $\begin{array}{l}\text { Marching Cubes } \\
+ \text { inner surface removal }\end{array}$ & Delaunay triangulation \\
\hline $\begin{array}{l}\hat{S}_{N R}: \text { Normalised results } \\
\text { for comparison }\end{array}$ & Outer surface of $F_{R}$ & Front surface of $D_{I}$ \\
\hline $\begin{array}{l}F_{C}: \text { Validation metric to } \\
\text { compare } \hat{S}_{M} \text { and } \hat{S}_{N R}\end{array}$ & $\begin{array}{l}\text { ICP } \\
\text { point-to-surface distance }\end{array}$ & $\begin{array}{l}\text { ICP } \\
\text { point-to-surface distance }\end{array}$ \\
\hline $\begin{array}{l}O_{C}: \text { Discrepancy between } \hat{S}_{M} \\
\text { and } \hat{S}_{N R} \text { computed by } F_{C}\end{array}$ & $\begin{array}{l}\text { i.median } \\
\text { i.stddev } \\
\text { i.s }, \text { max }^{\mathrm{ab}} \\
S_{R}\end{array}$ & $\begin{array}{l}\text { i.median } \\
\text { i.stddev } \\
\text { ab }, \text { i. }_{R} S_{R} \\
\end{array}$ \\
\hline$\overline{O_{Q I}}$ :Quality index from $O_{C}$ & $\operatorname{Mean}^{\mathrm{a}}, \operatorname{stddev}^{\mathrm{a}}, \max ^{\mathrm{a}}, \min ^{\mathrm{a}}$ & $\operatorname{Mean}^{\mathrm{a}}$, stddev $^{\mathrm{a}}, \max ^{\mathrm{a}}, \min ^{\mathrm{a}}$ \\
\hline$\overline{F_{H}}:$ Statistical tests & Wilcoxon test, ANOVA & Wilcoxon test, ANOVA \\
\hline
\end{tabular}

a in millimetres.

b i.* stands for results of ICP. 
Input parameters: Influence of the following input parameters (referred to as $P_{I}$ in Table 1) on method performance was studied:

- FOV type: mat, bright. The bright FOV is obtained by covering the skull phantom with gel.

- Light: weak, average, strong. The amount of light was measured using luminance histogram computed from the images.

- Focus of the microscope: minimal focus $\left(f_{\min }\right)$, maximal focus $\left(f_{\max }\right)$. These two focus values corresponded to the two extrema values of possible microscope focus settings.

Reference 1: For the first procedure, FIDSCAN, the skull phantom had a high resolution CT scan, with 8 fiducial markers attached. The positions of these markers were identified in the CT scan images and localised with the tracking system pointer in the neuronavigator's coordinate system. The rigid transformation computed between image and physical space was subsequently applied to the CT scan volume. To obtain an estimate of the registration quality, the Target Registration Error TRE [9] was computed over the surface of the skull phantom. The surface of the registered CT scan volume was then extracted using a Marching Cubes algorithm. Since the skull had thickness, the extracted surface was made up of an inner and an outer surface. To be comparable with reconstructed surface meshes, Reference 1 (referred to as $\hat{S}_{S N R}$ in Table 1) was defined as the outer surface of the skull phantom.

Reference 2: For the second procedure, LOCCLOUD, a cloud of 2,000 points was acquired on the skull phantom surface with the pointer. This points cloud was directly expressed into the neuronavigator's coordinate system. Reference 2 was the cloud which was triangulated by the Delaunay method in order to be compared to the reconstructed surface meshes.

Reconstructed surface meshes used for validation: For each of the 12 combinations of $P_{I}$ ( 2 FOV type levels $\times 3$ Light levels $\times 2$ Focus values $), 25$ image pairs were acquired, i.e., 25 surface meshes were intended to be reconstructed, with a size of $5 \times 3 \mathrm{~cm}$ (obtained for $f_{\min }$ ) and $8 \times 6 \mathrm{~cm}$ (obtained for $f_{\max }$ ). These 300 image pairs were acquired from different viewpoints, moving the microscope to cover all the facial part of the skull. In fact 8 surfaces (for $f_{\max }$ ) were not reconstructed. For the FIDSCAN procedure, the 292 reconstructed surface meshes were studied while in LOCCLOUD only 156 meshes which had a covering rate of $100 \%$ with $\hat{S}_{N R}$ (see Table 1) were manually selected.

Validation metric: For the two FIDSCAN and LOCCLOUD procedures the validation metric was the distance in millimetres from each vertex of a mesh (more than 30,000 vertices per mesh) to the nearest $\hat{S}_{N R}$ surface point (see Table 1). This was achieved using the Iterative Closest Point (ICP) algorithm. 
Discrepancy: For each reconstructed surface mesh, ICP was performed with Reference 1 or 2 as the target. As ICP results for one mesh, we kept the median (referred to as i.median), standard deviation (referred to as i.stddev) and maximal value (noted as i.max). We also defined the success ratio $S_{R}$ as the percentage of points reconstructed at a correct location compared to the total number of points, expressed by $S_{R}=\frac{R_{R} * Q_{R}}{100}$ where: $S_{R}$ stands for success ratio (as a percentage); $R_{R}$ stands for the reconstruction ratio (as a percentage), i.e., the number of points which have been reconstructed compared to the total number of points (pixels) studied; $Q_{R}$ stands for the quality ratio, i.e., the number of points of the reconstructed surface mesh being reconstructed under a threshold compared to the total number of points of the reconstruction. The threshold was determined as the worst i.median.

Quality indices: Quality indices were defined as follow for the three procedures: Precision of our 3D surface localiser is given by standard deviation and the maximal value of i.median, i.max and i.stddev. Accuracy is represented by the mean of the different values of i.median. Robustness is shown by the results obtained for the worst parameter combination and the success ratio $S_{R}$. Parameter influence is checked by a variance analysis of i.mean and $R_{R}$.

\section{Results}

Parameter influence. Only 292 surface meshes were reconstructed from 300 image pairs. This failure rate of $3 \%$ was only observed for the acquisitions with $f_{\max }$. To study parameter influence, reconstructed surface meshes were grouped by combining parameters into 12 samples. The distribution of median values i.median is shown in Figure 2 for both FIDSCAN and LOCCLOUD procedures. Parameter influence was not related to the procedure. Analysis of variance (ANOVA) of the mean distance i.mean was performed on the samples grouped by focus. Residuals were Gaussian, and the p-value was within $10^{-16}$. Consequently, the high impact of focus on accuracy was assumed. The same ANOVA was performed on the $f_{\text {min }}$ samples to test the impact of FOV type and luminance level. These two parameters have no significant impact on accuracy (pvalue $>0.5)$. The reconstruction ratio $R_{R}$ was also studied: only the luminance level and FOV type have an impact on $R_{R}$.

Results of the FIDSCAN procedure. The values of i.median, computed for over 30,000 vertices for each of the 150 reconstructed surface meshes with $f_{\text {min }}$, have a mean of $1.88 \pm 0.65 \mathrm{~mm}$ with a $\mathrm{Q} 75$ of $2.30 \mathrm{~mm}$ and a maximal value of $5.4 \mathrm{~mm}$. The mean of i.RMS is $3.32 \pm 1.20 \mathrm{~mm}$. The mean TRE was $4 \mathrm{~mm}$ on the front part of the skull, where the image pairs were acquired. A Wilcoxon non-parametric test showed that the Q95 of i.RMS for all focus values can be considered as below the TRE. The success ratio $S_{R}$ for all the focus had a mean of $62.15 \%$ with a threshold of $5 \mathrm{~mm}$. The minimum of $S_{R}$ was $24.38 \%$, however 219 out of 292 reconstructed surface meshes had a $S_{R}$ higher than $55 \%$. The best result of $84.15 \%$ was obtained for a bright FOV type and strong luminance. 


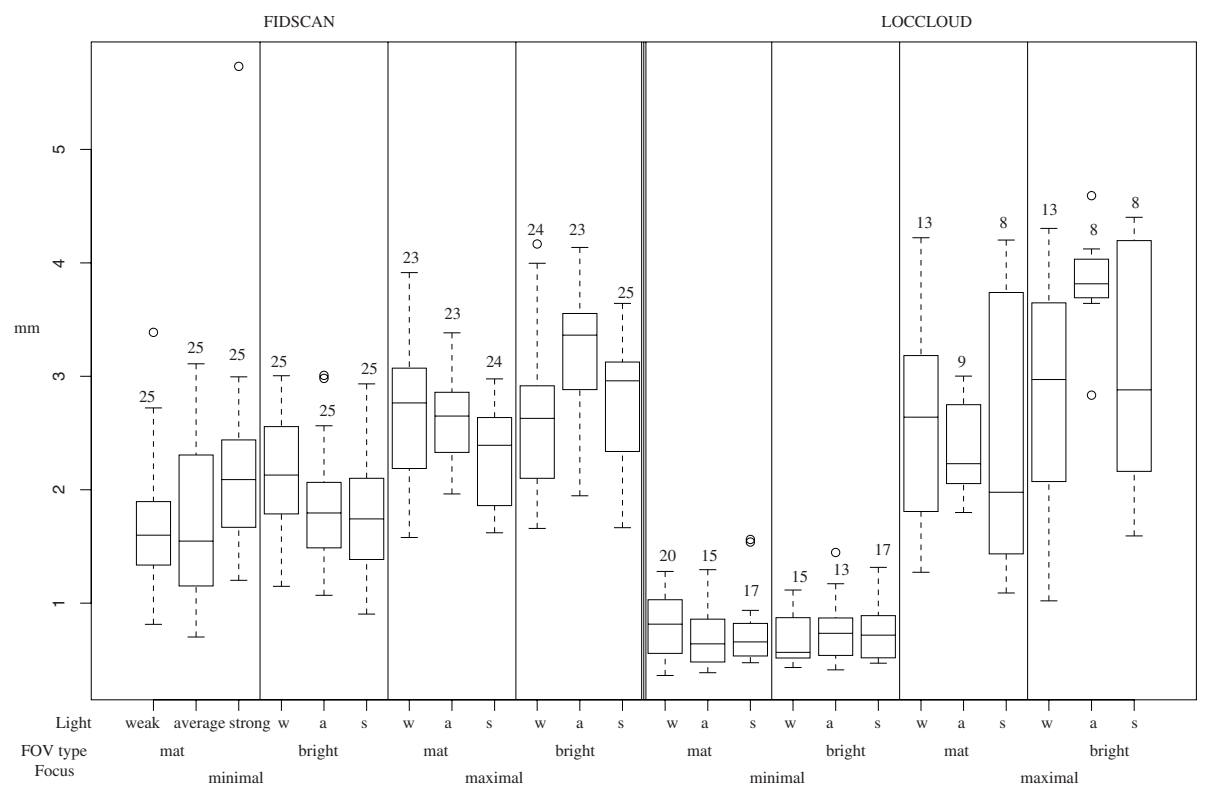

Fig. 2. Distribution of the median of the distance between reconstructed surface mesh and the reference surface for each sample corresponding to a parameters combination, for both procedures. The number of data in each sample is indicated by the number above its box-plot.

Results of the LOCCLOUD procedure. The mean of i.median for all 156 reconstructed surface meshes was $1.53 \pm 1.20 \mathrm{~mm}$. The results from LOCCLOUD with $f_{\max }$ are not significant since there were too few $3 \mathrm{D}$ surface meshes per sample (see Figure 21). However, the results seem to show no difference between FIDSCAN and LOCCLOUD procedures with $f_{\max }$, which might be explained by the limitations of the ICP validation metric. Considering the results for $f_{\min }$ alone, the mean of i.median was $0.74 \pm 0.27 \mathrm{~mm}$ with a maximal value of $1.56 \mathrm{~mm}$. A Wilcoxon test showed that the median can be expected to be within $1 \mathrm{~mm}$ (pvalue $\left.10^{-11}\right)$.

\section{Discussion}

Performance shown by the FIDSCAN procedure included the registration error of the reference surface, estimated by TRE. Therefore, this performance corresponded to a global error, which has been shown to be quite similar to the system error without the 3D surface localiser. In order to check the influence of the image-to-physical registration error on overall 3D surface localiser performance, the FIDSCAN procedure was repeated on the 292 reconstructed surface meshes, by just changing the image-to-physical registration method. The CT scan volume was registered using both fiducials and a surface described by a 2,000 points cloud acquired with the localisation pointer. In [10], this type of 
registration using a combination of fiducials and surface has been shown to have an error lower than the fiducial-based registration. Our results were consistent since the global error was reduced: the mean value of the i.median for the 150 reconstructed surface meshes with $f_{\min }$ was $1.41 \pm 0.65 \mathrm{~mm}$, in comparison with $1.88 \pm 0.65 \mathrm{~mm}$, i.e., a performance improvement of $25 \%$. The inherent error of our 3D surface localiser, which was estimated by the accuracy obtained using the LOCCLOUD procedure, was within $1 \mathrm{~mm}$. The success ratio $S_{R}$ was more than $55 \%$ for all the reconstructed surface meshes, which demonstrates the robustness of our method. Besides, the similar distribution of samples grouped by focus has shown good repeatability. Precision was given by the standard deviation of results and was within $1 \mathrm{~mm}$.

Because of the method point mapping step, based on ZNSSD, the luminance was expected not to have impact. The impact of focus values on performance can be explained by computation of the disparity interval in our method. For the minimal focus value, the computed interval was $[-40,40]$ whereas for a maximal focus value it was $[-45,12]$. This aspect of our method leaves room for improvement. However, stereoscopic systems are known to be sensitive to distance.

The fact that local deformations are respected by our 3D surface localiser needs highlighting, given that in [5] the reconstructed 3D surface is fitted to a spherical model. Moreover, local zones which are too dark or have too high specularity do not interfere with the rest of the surface reconstruction, unlike [4]. Studying two performance evaluation procedures allowed to emphasise the different sources of error. Moreover, while in [5] accuracy has been studied with 5 points of one reconstructed surface mesh, our performance evaluation relates to more than 30,000 points for each of the 156 to 292 reconstructed surface meshes.

Some limitations in the performance evaluation require underlining. Firstly, a clinical evaluation would probably be worse than evaluation on a phantom. The $3 \mathrm{D}$ surface localiser was tested for 3 clinical cases, but only the reconstruction ratio $R_{R}$ was computable, since there was no available reference surface. Results with skin, bone, or cortex images were similar to those computed on a phantom. In one clinical case, the reconstructed surface mesh of the skin was compared to the skin surface segmented from a preoperative MRI, and was rigidly registered to the physical space. The results obtained were a promising start: $3.25 \pm 1.58 \mathrm{~mm}$ with a Q75 of $6 \mathrm{~mm}$. The second limitation of the 3D surface localiser performance evaluation is that the ICP validation metric might over-evaluate performance, due to the fact that ICP does not give a symmetrical distance, and the homologous point in the reference surface of a reconstructed surface mesh point is supposed to be the nearest point from an Euclidean viewpoint.

\section{Conclusion}

To conclude, 3D surface localiser performance has been described and demonstrated using a rigorous procedure, which allowed differentiation between overall system error and the inherent error of the 3D surface localiser. This study is a first step towards validation. Indeed, we distinguish between validation and performance evaluation as follows: performance evaluation consists of computing quality indices whereas validation includes analysis of the evaluation results 
according to specific clinical contexts and objectives [7. Since our aim is the visualisation and the quantification of the cortical surface deformations, some thresholds would require defining. Visualisation should consist of enhanced virtuality by merging data from the real world (i.e., textured reconstructed surface meshes) and the virtual world. Surface deformation quantification could be used to update the preoperative structures near the surface, or as a constraint for volume approaches (e.g., 3D Ultrasounds). Indeed, further deep structures are subject to deformations which are difficult to predict from surface deformations alone [11].

Acknowledgements. The authors would like to thank Dr. Xavier Morandi for the fruitful discussions about the clinical relevance of the system and for the preliminary clinical results.

\section{References}

1. Edwards PJ et al. Design and evaluation of a system for microscope-assisted guided interventions (MAGI). IEEE Trans Med Imaging, 19(11):1082-93, 2000.

2. Jannin P, Bouliou A, Journet E, and Scarabin JM. A ray-traced texture mapping for enhanced virtuality in image-guided neurosurgery. Stud Health Technol Inform, 29:553-63, 1996.

3. Fleig OJ, Devernay F, Scarabin JM, and Jannin P. Surface reconstruction of the surgical field from stereoscopic microscope views in neurosurgery. In Proc. CARS'2001, pages 259-64, 2001.

4. Skrinjar O, Tagare H, and Duncan J. Surface growing from stereo images. In IEEE CVPR'00, volume II, pages 571-76, 2000.

5. Sun $\mathrm{H}$ et al. Estimating cortical surface motion using stereopsis for brain deformation models. In Proc. MICCAI'03, pages 794-801. LNCS 2878.

6. Audette M, Siddiqi K, and Peters T. Level-set surface segmentation and fast cortical range image tracking for computing intrasurgical deformations. In Proc. MICCAI'99, pages 788-97. LNCS 1679.

7. Jannin $\mathrm{P}$ et al. Validation of medical image processing in image-guided therapy. IEEE Trans Med Imaging, 21(11):1445-49, 2002.

8. Devernay F and Faugeras O. From projective to euclidean reconstruction. In IEEE CVPR'96, pages 264-69, 1996.

9. Fitzpatrick JM, West JB, and Maurer CR. Predicting error in rigid-body, pointbased registration. IEEE Trans Med Imaging, 17(5):694-702, 1998.

10. Maurer CR, Maciunas RJ, and Fitzpatrick JM. Registration of head CT images to physical space using a weighted combination of points and surfaces. IEEE Trans Med Imaging, 17(5):753-61, 1998.

11. Hartkens $\mathrm{T}$ et al. Measurement and analysis of brain deformation during neurosurgery. IEEE Trans Med Imaging, 22(1):88-92, 2003. 